\title{
CDDO-Me Elicits Anti-Breast Cancer Activity by Targeting LRP6 and FZD7 Receptor Complex ${ }^{\llbracket}$
}

\author{
Liang Zhou, ${ }^{1}$ Zhongyuan Wang, ${ }^{1}$ Shubin Yu, Yanpeng Xiong, Jiaoyang Fan, Yansi Lyu, \\ Zijie Su, Jiaxing Song, Shanshan Liu, Qi Sun, and Desheng Lu
}

Guangdong Key Laboratory for Genome Stability \& Disease Prevention, Carson International Cancer Center, Department of Pharmacology, Shenzhen University Health Science Center, Shenzhen, Guangdong, China (L.Z., Z.W., S.Y., Y.X., J.F., Z.S., J.S., S.L., Q.S., D.L.) and Department of Dermatology, Shenzhen University General Hospital, Shenzhen, Guangdong, China (Y.L.)

Received October 28, 2019; accepted January 21, 2020

\begin{abstract}
Aberrant activation of the $\mathrm{Wnt} / \beta$-catenin pathway leads to the development of multiple cancers, including breast cancer. Development of therapeutic agents against this signaling pathway is an urgent need. In this study, we found that 2-cyano-3, 12-dioxooleana-1, 9(11)-dien-28-oic acid-methyl ester (CDDO-Me) could inhibit Wnt/ $\beta$-catenin signaling mainly through targeting the low-density lipoprotein receptor-related protein (LRP) 6 and Frizzled (FZD) 7 receptor complex. This compound induced the degradation and ubiquitination of LRP6 and Fzd7 via the lysosomal pathway. We further showed that CDDO-Me mediated the degradation of FZD7 in an LRP6 ectodomain-dependent manner. In breast cancer cells, treatment with CDDO-Me increased the degradation of LRP6 and FZD7 and reduced the levels of phosphorylated Disheveled (DVL) 2 and active $\beta$-catenin, resulting in the downregulation of Wnt target genes and several cancer stem cell (CSC) marker genes. In a murine xenograft bearing mouse mammary tumor virus (MMTV)-Wnt1-driven mammary tumor, administration of CDDO-Me significantly inhibited tumor growth and was accompanied by reduced expression of
\end{abstract}

phosphorylated and total LRP6, phosphorylated and unphosphorylated DVL2, active $\beta$-catenin, several Wnt target genes, and CSC marker genes. Collectively, the results of our study present that CDDO-Me is a potent $\mathrm{Wnt} / \beta$-catenin signaling inhibitor that may be a promising therapeutic agent against breast cancer.

\section{SIGNIFICANCE STATEMENT}

Blocking the membrane receptor complex consisting of lowdensity lipoprotein receptor-related protein (LRP) 6 and Frizzled (FZD) 7 may help developing therapeutic approaches for cancers, including breast cancers. Our study indicates that 2-cyano-3, 12-dioxooleana-1, 9(11)-dien-28-oic acid-methyl ester (CDDO-Me) can inhibit Wnt/ $\beta$-catenin signaling by inducing the ubiquitination and degradation of LRP6/FZD7 membrane receptor complex via a lysosomal pathway. We also found that the ectodomain of LRP6 is essential for CDDOMe-induced FZD7 degradation. Defining CDDO-Me as a novel inhibitor of Wnt/ $\beta$-catenin signaling, our results provide insight into the mechanism of its anticancer activity.

\section{Introduction}

The Wnt/ $\beta$-catenin-signaling cascade is a highly conserved pathway and essential for embryonic development, stem cell maintenance, and tissue homeostasis. The dysregulation of

This work was supported by the National Nature Science Foundation of China (Grants 31870754, 31970739, and 31501143), the Nature Science Foundation of Guangdong Province (Grant 2014A030310168), the Shenzhen Peacock Innovation Team Project (Grant KQTD20140630100658078), the Shenzhen Peacock Plan (Grants 827000183 and 827000186), the Shenzhen Basic Research Program (Grants JCYJ20150525092941006, JCYJ20170302143447936, and JCYJ20170817094611664), and SZU Medical Young Scientists Program (71201-000001).

${ }^{1}$ L.Z. and Z.W. contributed equally to this work.

https://doi.org/10.1124/jpet.119.263434.

S This article has supplemental material available at jpet.aspetjournals.org the $\mathrm{Wnt} / \beta$-catenin pathway by mutations and epigenetic changes is frequently found in various human cancers, including breast cancer (MacDonald et al., 2009; Clevers and Nusse, 2012; Nusse and Clevers, 2017; Krishnamurthy and Kurzrock, 2018). The transcriptional coactivator $\beta$-catenin is a core component of the pathway, whose protein level and activity are closely controlled by a destruction complex in the cytoplasm. This complex is composed of the scaffolding protein AXIN, the adenomatous polyposis coli protein, casein kinase $1 \alpha$, glycogen synthase kinase $3 \beta$ (GSK3 $\beta$ ), and $\beta$-transducin repeats-containing proteins. Wnt $/ \beta$-catenin signaling is initiated by binding of Wnt ligands to their transmembrane receptors of the Frizzled (FZD) family and the low-density lipoprotein receptor-related protein (LRP) 5/6. Subsequently,

ABBREVIATIONS: BrdU, bromodeoxyuridine; CDDO, 2-cyano-3, 12-dioxooleana-1, 9(11)-dien-28-oic acid; CDDO-Im, CDDO-imidazolide; CDDOMe, CDDO-methyl ester; CSC, cancer stem cell; DVL, Disheveled; EA, ethacrynic acid; FZD, Frizzled; $\beta$-gal, $\beta$-galactosidase; GSK3 $\beta$, glycogen synthase kinase $3 \beta$; HEK, human embryonic kidney; JAK, Janus-activated kinase; LGR5, leucine-rich repeat-containing G-protein-coupled receptor 5; LRP, low-density lipoprotein receptor-related protein; LRP6 $\mathrm{N}$, LRP6 mutant lacking the N-terminal extracellular domain; Luc, luciferase; MG132, carbobenzoxy-L-leucyl-L-leucyl-L-leucinal; MMTV, mouse mammary tumor virus; NAC, N-acetyl-L-cysteine; NF- $\kappa$ B, nuclear factor- $\kappa \mathrm{B}$; OA, oleanic acid; Oct4, octamer-binding transcription factor 4; PCR, polymerase chain reaction; shRNA, short hairpin RNA; STAT, signal transducer and activator of transcription; Ub, ubiquitin; Wnt3A-CM, Wnt3A-conditioned medium; YAP, yes-associated protein 1. 
casein kinase $1 \alpha$ is activated and phosphorylates Disheveled (DVL). Receptor complexes further induce the formation of LRP6 signalosomes, resulting in the phosphorylation of LRP6 at multiple sites via distinct kinases, which triggers the downstream cascade that leads to $\beta$-catenin stabilization. Finally, free $\beta$-catenin accumulates in the cytoplasm, which allows it to translocate to the nucleus, and then $\beta$-catenin interacts with the T-cell factor/lymphoid-enhancing factor transcription factors to initiate transcription of Wnt target genes (MacDonald et al., 2009; Clevers and Nusse, 2012).

Triterpenoids are a large family of structurally related compounds biosynthesized in some plants by the cyclization of squalene. Oleanolic acid (OA) (3b-hydroxyolean-12-en-28oic acid) is a naturally occurring triterpenoid that exhibits antioxidant, antibacterial, antifungal, anticancer, and antiinflammatory activities (Supplemental Fig. 1A) (Ayeleso et al., 2017). To improve the pharmacological efficacy of OA, syntheses of a series of novel derivatives have been conducted, including 2-cyano-3, 12-dioxooleana-1, 9(11)-dien-28-oic acid (CDDO), CDDO-imidazolide (CDDO-Im), and CDDO-methyl ester (CDDO-Me) (Supplemental Fig. 1A). Among them, CDDO-Me displays potent anticancer activity in the treatment of multiple types of cancer in preclinical and clinical studies (Wang et al., 2017). CDDO-Me could induce cytotoxicity in different cancer cells, including ovarian cancer, prostate cancer, leukemia, breast cancer, lung cancer, and pancreatic cancer, but with less toxicity in normal cells (Wang et al., 2014b). Accumulating evidence demonstrates that CDDO-Me is a multitargeting molecule. This compound has been shown to target nuclear factor erythroid 2-related factor 2, prosurvival phosphatidylinositol-3-kinase/ protein kinase $\mathrm{B} /$ mammalian target of rapamycin, JAK/STAT, mitogen-activated protein kinase/extracellular signal-regulated kinases 1 and 2, nuclear factor $-\kappa \mathrm{B}(\mathrm{NF}-\kappa \mathrm{B})$, and peroxisome proliferator-activated receptor $\gamma$ signaling pathways (Ahmad et al., 2008; Deeb et al., 2009; Yadav et al., 2010; Ai et al., 2015; To et al., 2015; Wang et al., 2015, 2017; Xu et al., 2017; Zagorski et al., 2018). However, the molecular mechanism underlying the anticancer activity of CDDO-Me remains unclear.

In this study, we illustrate that CDDO-Me is a potent Wnt/ $\beta$-catenin signaling antagonist. This compound could directly bind to the extracellular domain of LRP6 and induce lysosomal degradation of LRP6/FZD7 receptor complex, resulting in a decrease in DVL phosphorylation and the downregulation of active $\beta$-catenin and total $\beta$-catenin protein. In a xenograft model of mouse mammary tumor
A

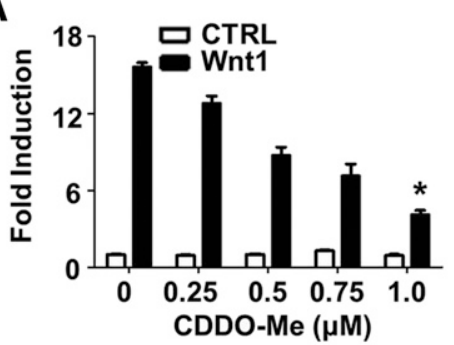

D

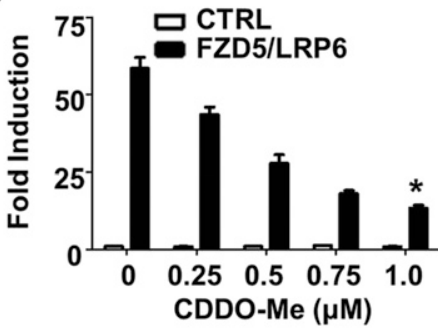

G

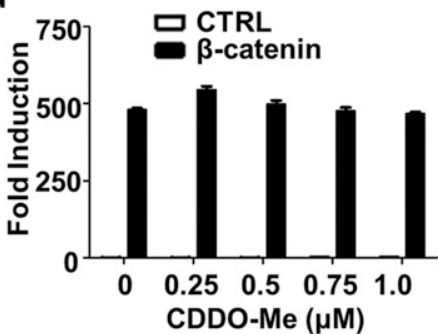

B

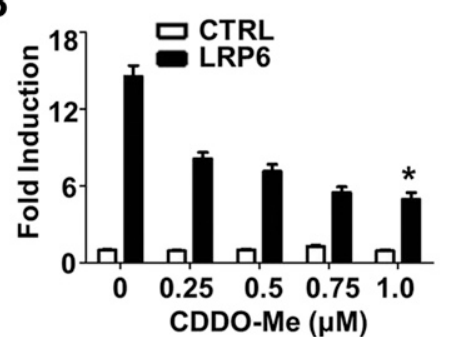

E

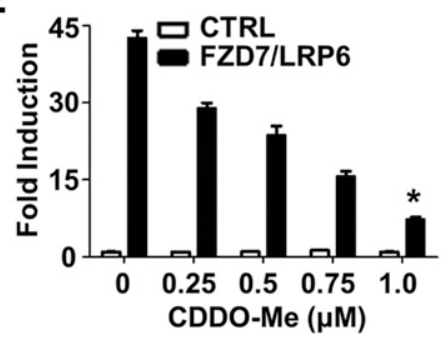

H

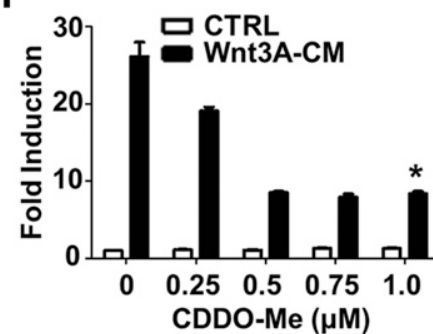

C

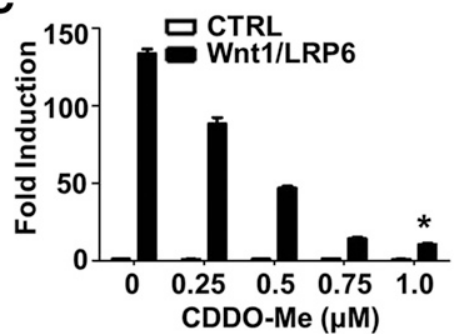

$\mathbf{F}$

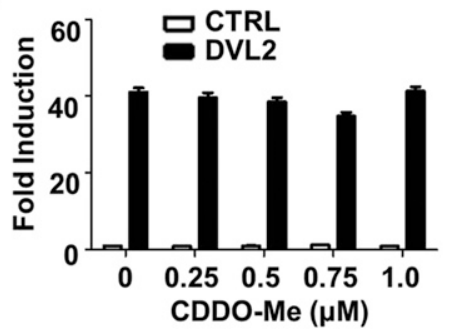

I

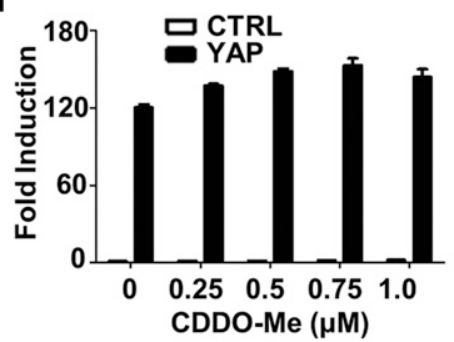

Fig. 1. CDDO-Me specifically inhibits the Wnt/ $\beta$-catenin signaling pathway. (A-G) The SuperTOPFlash reporter was transfected into HEK293T cells together with empty vector or expression plasmids encoding Wnt1 (A), LRP6 (B), Wnt1 and LRP6 (C), LRP6 and FZD5 (D), LRP6 and FZD7 (E), DVL2 $(\mathrm{F})$, and $\beta$-catenin (G). (H) The SuperTOPFlash reporter gene was transfected into HEK293T cells, after which the cells were treated with control-CM or Wnt3A-CM. (I) HEK293T cells were transfected with an $8 \times$ GTIIC-Luc reporter along with empty vector or YAP expression plasmid. The transfected cells were incubated with vehicle or the indicated concentrations of CDDO-Me for 24 hours. The cells were then lysed, and the luciferase activities were examined and normalized according to $\beta$-gal values. Each treatment was performed in six replicates. The values are mean \pm S.E.M. Statistical analysis was conducted using one-way ANOVA followed by a Dunnett- $t$ test $(\mathrm{A}-\mathrm{I})$. CTRL, control. $* P<0.05$ compared with the vehicle control. 
virus(MMTV)-Wnt1-derived cancer cells, administration of CDDO-Me significantly suppressed tumor growth with a concomitant inhibition of the Wnt/ $\beta$-catenin signaling cascade.

\section{Materials and Methods}

Reagents and Plasmids. Oleanolic acid and $N$-acetyl-L-cysteine (NAC) and was purchased from Sigma-Aldrich (Sigma-Aldrich, MO). CDDO and CDDO-Me were obtained from MedChemExpress (MCE, NJ). A Food and Drug Administration-approved drug library was purchased from MedChemExpress (MCE). The reporter plasmids SuperTOPFlash and $8 \times$ GTIIC-Luc and the expression plasmids encoding Wnt1, LRP6, LRP6 mutant lacking the $N$-terminal extracellular domain (LRP6 $\Delta$ N), FZD5, FZD7, DVL2, $\beta$-catenin, YAP, and $\beta$-gal have been described previously (Wang et al., 2016; Su et al., 2018).

Cell Culture. Human embryonic kidney (HEK) 293T, MDA-MB231, MDA-MB-468, and Hs578T cells were obtained from the typical culture preservation commission cell bank of the Chinese Academy of Sciences in China. Mouse fibroblast L-cells, L-cells stably transfected with Wnt3A (L-Wnt3A), were obtained from the American Type Culture Collection (ATCC, Manassas, VA). HEK293T, L-, L-Wnt3A, and Hs578T cells were cultured in Dulbecco's modified Eagle's medium supplemented with $10 \%$ FBS (Gibco, TN) and $1 \%$ penicillinstreptomycin (Gibco) in a humidified incubator at $37^{\circ} \mathrm{C}$ with $5 \% \mathrm{CO}_{2}$.
As described previously, Wnt3A-conditioned medium (Wnt3A-CM) and control-conditioned medium were prepared (Willert et al., 1999). MDA-MB-231 and MDA-MB-468 cells were maintained in Leibovitz's L-15 medium (Gibco) supplemented with $10 \%$ FBS and $1 \%$ penicillinstreptomycin in a humidified incubator at $37^{\circ} \mathrm{C}$ without $\mathrm{CO}_{2}$.

Luciferase Reporter Assays. HEK293T cells were cultured in 24-well plates and then transiently transfected using LipofectAMINE 2000 (Invitrogen, CA) in sixtuplicate with $0.25 \mu \mathrm{g}$ of luciferase reporter plasmid, $50 \mathrm{ng}$ of control plasmid for $\beta$-gal, and 50-200 ng of the indicated expression vectors according to the manufacturer's instructions. After 24 hours, the cells were incubated with the indicated concentrations of compounds. In some experiments, cells were treated with Wnt3A-CM or control-conditioned medium together with the compounds, as indicated in the figure legends. Luciferase activity was measured with the Luciferase Assay System (Promega, WI) and normalized using $\beta$-gal activity as an internal control.

Immunoblot Analyses. Protein samples were extracted from cells or tumor tissues using lysis buffer containing $20 \mathrm{mM}$ Tris $\cdot \mathrm{HCl}$, pH 7.4; 150 mM NaCl; 1 mM EDTA; 1 mM EGTA; 1\% Triton X-100; protease inhibitor cocktail (HY-K0010; MedChemExpress); phosphatase inhibitor cocktail (HY-K0021; MedChemExpress); and $1 \mathrm{mM}$ phenylmethylsulfonyl fluoride. Equal amounts of proteins were size fractionated by $6 \%-12 \%$ SDS-PAGE and electroblotted onto polyvinylidene fluoride membranes (GE healthcare, PA). After incubation with the primary antibodies at $4^{\circ} \mathrm{C}$ overnight, the blots were detected with relevant horseradish peroxidase-conjugated secondary antibody (ThermoFisher, MA) and ECL Plus Western Blotting Substrate

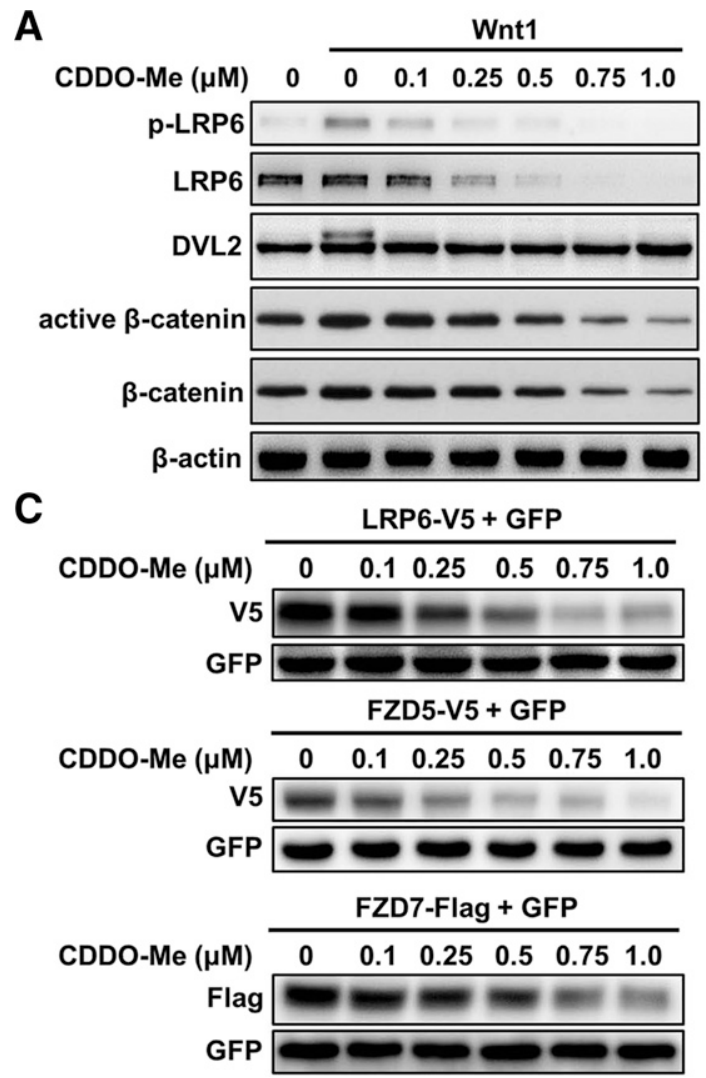

B

Wnt3A-CM

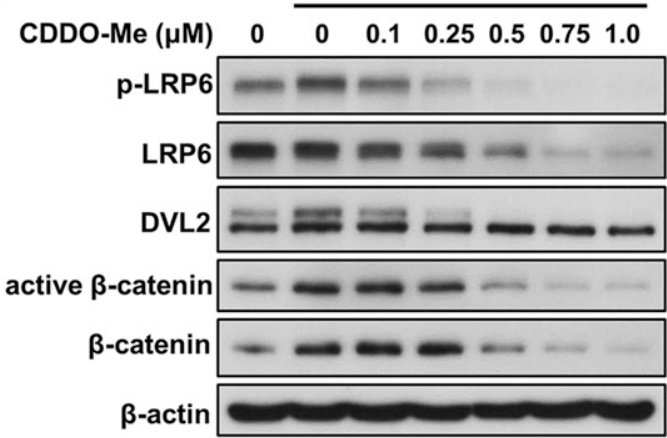

Fig. 2. CDDO-Me inhibits Wnt/ $\beta$-catenin signaling in HEK293T cells. (A) HEK293T cells were transfected with empty vector or Wnt1 expression plasmid, and then the cells were treated with the indicated concentrations of CDDO-Me for 24 hours. Phosphorylated LRP6 (p-LRP6) (Ser1490), total LRP6, DVL2, active $\beta$-catenin, and total $\beta$-catenin were detected by immunoblotting. The phosphorylated DVL2 showed slower mobility after SDSPAGE. (B) HEK293T cells were treated with vehicle or the indicated concentrations of CDDO-Me in the absence or presence of Wnt3A-CM for 24 hours. Phosphorylated LRP6 (Ser1490), total LRP6, DVL2, active $\beta$-catenin, and total $\beta$-catenin were detected by immunoblotting. The phosphorylated DVL2 showed slower mobility after SDS-PAGE. (C) HEK293T cells were transfected with the expression plasmids for LRP6-V5 and GFP, FZD5-V5 and GFP, or FZD7-Flag and GFP. The cells were then treated with the indicated concentrations of CDDO-Me for 24 hours. Expression of exogenous proteins were detected by immunoblotting with the corresponding antitag antibodies. GFP was used to normalize the transfection efficiency. 
A

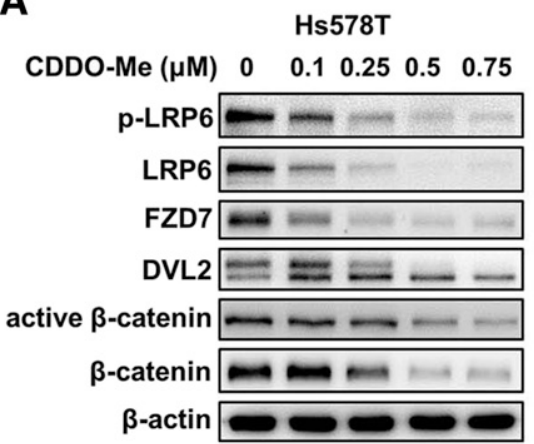

D

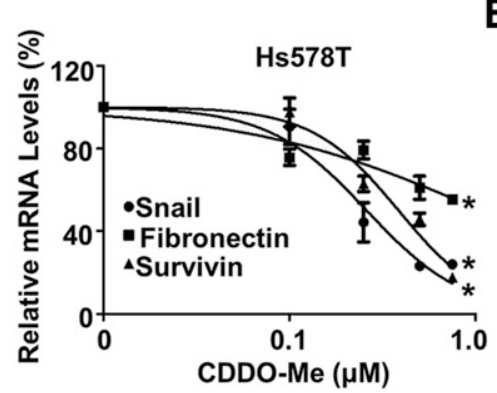

B

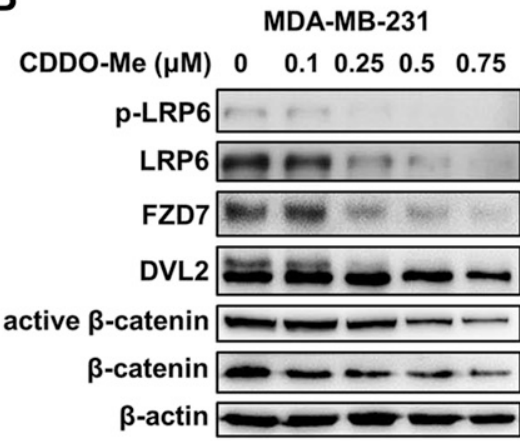

E

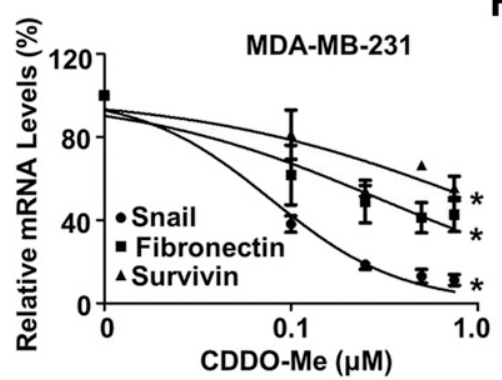

C

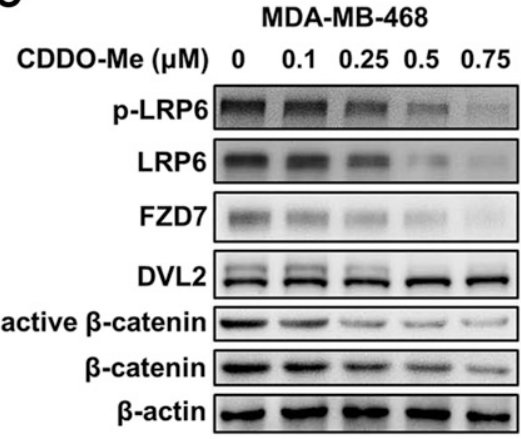

F

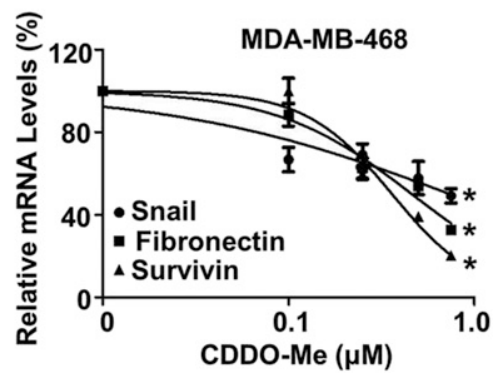

Fig. 3. CDDO-Me inhibits Wnt/ $\beta$-catenin signaling in breast cancer cells. (A-C) Hs578T (A), MDA-MB-231 (B), and MDA-MB-468 (C) cells were treated with the indicated concentrations of CDDO-Me for 24 hours. Phosphorylated LRP6 (p-LRP6) (Ser1490), total LRP6, FZD7, DVL2, active $\beta$-catenin, and total $\beta$-catenin were detected by immunoblotting. The phosphorylated DVL2 showed slower mobility after SDS-PAGE. (D-F) Hs578T (D), MDA-MB-231 (E), and MDA-MB-468 (F) cells were treated with the indicated concentrations of CDDO-Me for 24 hours. The Wnt target genes Snail, Fibronectin, and Survivin were detected by real-time PCR. The data from three independent experiments are presented. The values are mean \pm S.E.M. Statistical analysis was conducted using one-way ANOVA followed by a Dunnett- $t$ test (D-F). ${ }^{*} P<0.05$ compared with the vehicle control.

(ThermoFisher). Signals were visualized using either X-ray film or Tanon 5200 Chemiluminescent Imaging System (Tanon, Shanghai, China). The antibodies used were listed in Supplemental Table 1.

Ubiquitination Assays. HEK293T cells cotransfected with indicated expression plasmids were treated with the indicated concentrations of CDDO-Me for 24 hours. Cells were lysed with lysis buffer containing $20 \mathrm{mM}$ Tris $\cdot \mathrm{HCl}, \mathrm{pH} 7.4 ; 150 \mathrm{mM} \mathrm{NaCl} ; 1 \mathrm{mM}$ EDTA; $1 \mathrm{mM}$ EGTA; 1\% Triton X-100; protease inhibitor cocktail (HY-K0010; MedChemExpress); phosphatase inhibitor cocktail (HY-K0021; MedChemExpress); and $1 \mathrm{mM}$ phenylmethylsulfonyl fluoride after treatment with $10 \mu \mathrm{M}$ MG132 for 6 hours. The supernatant fractions were collected by centrifugation at 12,000 rpm for 15 minutes and subjected to immunoprecipitation using anti-V5 or anti-FLAG M2-Sepharose followed by Western blotting using specific antibodies as indicated. The antibodies used were listed in Supplemental Table 1.

Real-Time Polymerase Chain Reaction Analyses. Total RNA from cells or tumor tissues was isolated using RNAiso Plus Reagent (TaKaRa) and then reverse-transcribed into cDNA using the Primescript RT Reagent Kit (TaKaRa) according to the manufacturer's instructions. Real-time polymerase chain reaction (PCR) assays were performed using an ABI Prism 7300 Real-Time PCR System (Applied Biosystems, Foster City, CA) with $2 \times$ SYBR Green qPCR Master Mix (Promega). The comparative $\mathrm{Ct}$ method was used to analyze relative expression of genes. The primers were listed in Supplemental Table 2.

Colony Formation Assays. In medium containing 10\% FBS in six-well plates overnight, $1 \times 10^{3}$ cells/well were grown, and then the indicated concentrations of CDDO-Me were added into the medium. The cells were cultured in a humidified incubator at $37^{\circ} \mathrm{C}$ with $5 \% \mathrm{CO}_{2}$ for 10 days, and then the colonies were fixed with $4 \%$ paraformaldehyde, stained with $0.1 \%$ crystal violet, and photographed. Then the colonies were counted and quantified.

Cell Viability and Proliferation Assays. Cells were seeded onto 96 -well plates at a density of $2 \times 10^{4}$ cells/well and cultured overnight. The cells were then treated with DMSO or serially diluted CDDO-Me for 24 or 48 hours. For viability assay, the cells were incubated with

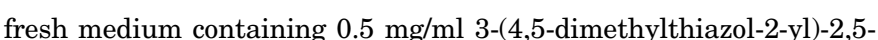
diphenyltetrazolium bromide for another 4 hours. Then the medium was removed, formazan crystals were dissolved in DMSO, and the absorbance was measured at $570 \mathrm{~nm}$. To examine the cell proliferation, the bromodeoxyuridine (BrdU) incorporation assay was carried out using the Cell Proliferation ELISA BrdU Chemiluminescent Kit (Roche) as directed by the manufacturer's instruction.

Immunofluorescence Analyses. FZD7-Alexa Fluor 594 antibody was generated by conjugating FZD7 antibody to Alexa Fluor 594 using Zenon Alexa Fluor 594 Human IgG Labeling Kit (Z25407; Invitrogen) according to the manufacturer's instructions. The cells were incubated with $0.1 \mu \mathrm{M}$ FZD7-Alexa Fluor 594 antibody for 30 minutes on ice, and after this they were washed twice with PBS. The cells were then treated with $0.4 \mu \mathrm{M}$ CDDO-Me for 3.5 hours in an incubator before the addition 60 nM LysoTracker Deep Red (L12492; Invitrogen) for 30 minutes. After fixing with $4 \%$ paraformaldehyde, 4',6-diamidino-2-phenylindole was used for visualization of cell nucleus. Sections were observed by using a Leica laser-scanning confocal microscope.

Sphere Formation Assays. Ultra-low attachment 24-well plates (Corning) were chosen to culture unattached spheroid cells. Hs578T cells were seeded as single cells into the sphere culture medium MammCult (STEMCELL technologies) supplemented with $0.48 \mu \mathrm{g} / \mathrm{ml}$ hydrocortisone and $4 \mu \mathrm{g} / \mathrm{ml}$ heparin in the presence or absence of the indicated amounts of CDDO-Me. After 10 days incubation, spheres with diameter over $50 \mu \mathrm{m}$ were counted, and representative fields were microphotographed.

Xenograft Mouse Model and Treatment with CDDO-Me. All animal experiments were performed according to the protocols approved by the Administrative Committee on Animal Research of Shenzhen University, permit AEWC-201412003 (approved on Dec. 25, 2014). MMTV-Wnt1 transgenic mice on an FVB background [FVB.Cg$\mathrm{Tg}(\mathrm{Wnt1}) 1 \mathrm{Hev} / \mathrm{J}]$ were purchased from Jackson Laboratories, and female BALB/c nude mice were obtained from Shanghai SLAC Laboratory Animal Co., Ltd. After the breast tumors grew to 
A

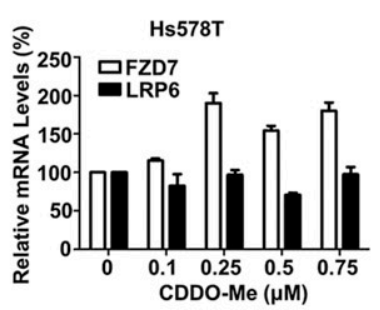

E

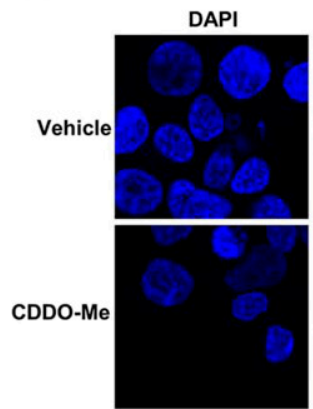

B

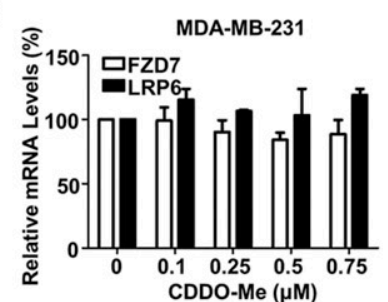

C

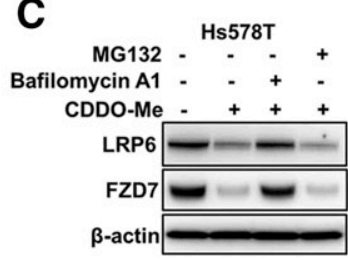

$\mathbf{F}$

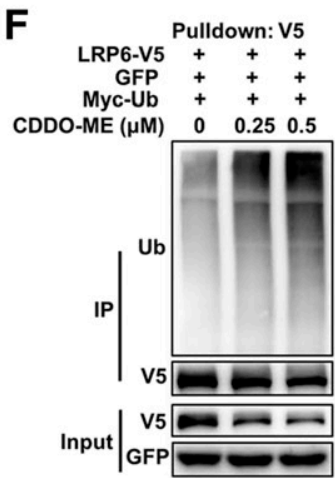

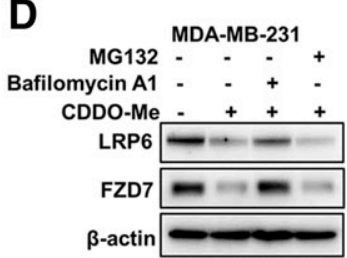

G \begin{tabular}{rccc}
\multicolumn{3}{c}{ Pulldown: Flag } \\
FZD7-Flag & + & + & + \\
GFP & + & + & + \\
Myc-Ub & + & + & + \\
CDDO-ME $(\mu \mathrm{M})$ & 0 & 0.25 & 0.5
\end{tabular}

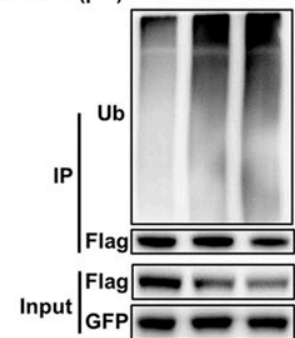

Fig. 4. CDDO-Me induces the degradation of LRP6/FZD7 receptor complex via a lysosome-dependent manner. (A and B) Hs578T (A) and MDA-MB-231 (B) cells were treated with the indicated concentrations of CDDO-Me for 24 hours. The relative mRNA levels of LRP6 and FZD7 were detected by realtime PCR. The data from three independent experiments are presented. (C and D) Hs578T (C) and MDA-MB-231 (D) cells were incubated with $0.4 \mu \mathrm{M}$ CDDO-Me for 16 hours before treatment with $20 \mathrm{nM}$ Bafilomycin A1 or $10 \mu \mathrm{M}$ MG132. After incubation for another 8 hours, the cells were harvested, and the protein levels of LRP6 and FZD7 were detected by immunoblotting. (E) HEK293T cells stably transfected with FZD7 were treated with $0.1 \mu$ M antiFZD7-Alexa Fluor 594 for 30 minutes on ice. The cells were then treated with vehicle or $0.4 \mu \mathrm{M}$ CDDO-Me for 3.5 hours before adding $60 \mathrm{nM}$ LysoTracker Deep Red. After incubation for another 30 minutes, the cells were fixed, and nucleus was stained with 4',6-diamidino-2-phenylindole (DAPI) (F and G) HEK293T cells cotransfected with expression plasmids for GFP, myc-Ub, and LRP6-V5 (F) or FZD7-Flag (G) were incubated with the indicated concentrations of CDDO-Me for 24 hours. Cell lysates were extracted and subjected to immunoprecipitation (IP) using anti-V5 (F) or anti-Flag M2-Sepharose (G), which was followed by Western blotting using specific antibodies as indicated. GFP was used to normalize the transfection efficiency. Scale bar, $20 \mu \mathrm{m}$.

$\sim 500 \mathrm{~mm}^{3}$, the MMTV-Wnt1 mice were sacrificed, and tumor fragments were implanted subcutaneously into BALB/c nude mice. When the tumors reached $\sim 50 \mathrm{~mm}^{3}$, the mice were randomly divided into two groups and treated by intraperitoneal injection with the vehicle (0.8\% DMSO/12\% Cremophor/8\% ethanol in normal saline) or $5 \mathrm{mg} / \mathrm{kg}$ CDDO-Me in vehicle twice weekly. Subsequently, tumor volumes were measured with a caliper and calculated using the following formula: $0.52 \times$ length $\times$ width $^{2}$. When tumor size reached $15 \mathrm{~mm}$ in diameter or tumors showed signs of ulceration, $\mathrm{CO}_{2}$ euthanasia was performed. The tumors were then excised, weighed, and photographed.

Histologic Analyses. The tumors were fixed in formalin, embedded with paraffin, and sectioned. Hematoxylin and eosin staining and immunohistochemistry analysis were carried out as previously described (Wang et al., 2016). The primary antibodies used were listed in Supplemental Table 1.

Statistical Analyses. Statistical analyses were carried out using Student's $t$ test or one-way ANOVA followed by a Dunnett- $t$ test. Results are presented as mean \pm S.E.M. A $P$ value less than 0.05 was considered statistically significant.

\section{Results}

Inhibition of Wnt/ $\beta$-Catenin Signaling by CDDO-Me. An initial screen of a Food and Drug Administration-approved drug library was performed in HEK293T cells using a 96-well plate-based SuperTOPFlash reporter system, in which Wnt3A-CM was used to activate the Wnt/ $\beta$-catenin pathway. The screen identified OA, CDDO, and CDDO-Me as antagonists of $\mathrm{Wnt} / \beta$-catenin signaling. Previous studies have identified these three compounds as nuclear factor erythroid 2-related factor 2 activators and NF- $\kappa \mathrm{B}$ inhibitors (Ahmad et al., 2006; To et al., 2015; Xu et al., 2017; Zagorski et al., 2018).
Concerning the importance of the Wnt/ $\beta$-catenin-signaling pathway in initiation and progression of various cancers (Nusse and Clevers, 2017; Mirabelli et al., 2019; Zhong and Virshup, 2020 ), we characterized the antagonistic effect of these three compounds on Wnt/ $\beta$-catenin signaling.

To prove the Wnt inhibitory effect of CDDO-Me, a SuperTOPFlash reporter plasmid was transfected into HEK293T cells along with Wnt1, LRP6, Wnt1/LRP6, FZD5/LRP6, FZD7/LRP6, DVL2, and $\beta$-catenin expression plasmids, respectively. CDDOMe dose-dependently inhibited the transcriptional activity of Wnt signaling activated by Wnt1, LRP6, Wnt1/LRP6, FZD5/ LRP6, and FZD7/LRP6 (Fig. 1, A-E). The increased transcriptional activity induced by the Wnt3A-CM was also blocked by CDDO-Me (Fig. 1H). However, CDDO-Me did not inhibit the SuperTOPFlash activity induced by either DVL2 or $\beta$-catenin (Fig. 1, F and G), suggesting that CDDO-Me may act on the upstream elements of DVL2 and $\beta$-catenin. In control experiments, CDDO-Me at Wnt inhibitory concentrations did not suppress YAP-mediated activation of a Hippo reporter $(8 \times$ GTIIC-Luc) (Fig. 1I), indicating that the Wnt inhibitory effect of CDDO-Me is specific.

We also evaluated the effects of CDDO-Me analogs CDDO and OA on Wnt/ $\beta$-catenin signaling using a SuperTOPFlash reporter assay. CDDO and $\mathrm{OA}$ inhibited Wnt signaling activated by Wnt1, LRP6, Wnt1/LRP6, FZD5/LRP6, and FZD7/LRP6 (Supplemental Fig. 1, B-K), although they did not suppress the transcriptional activity induced by either DVL2 or $\beta$-catenin (Supplemental Fig. 1, L-O). CDDO and OA also reduced Wnt3A-CM-induced activity of the SuperTOPFlash reporter in a dose-dependent manner (Supplemental Fig. 1, P and Q). As expected, both compounds did not inhibit 
A

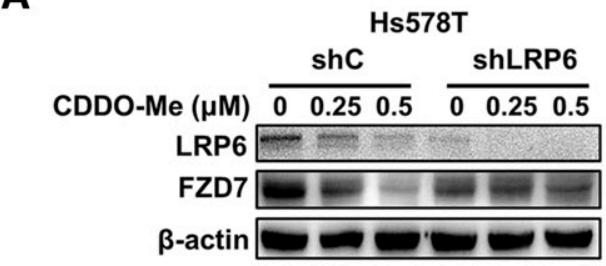

B

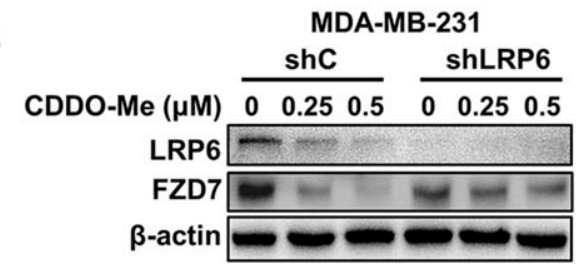

C
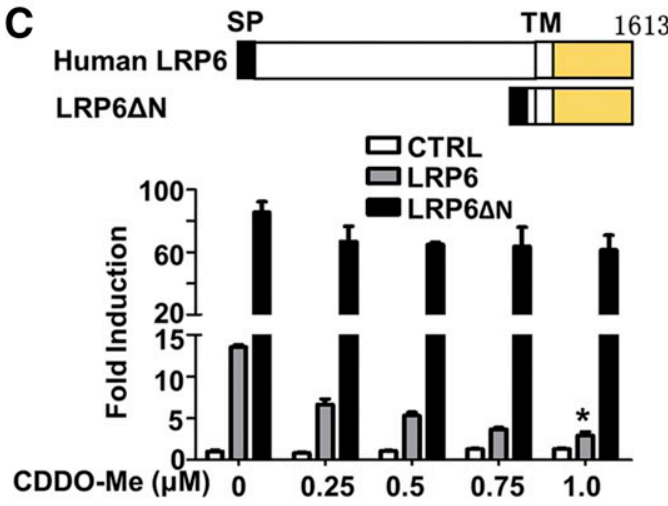

D

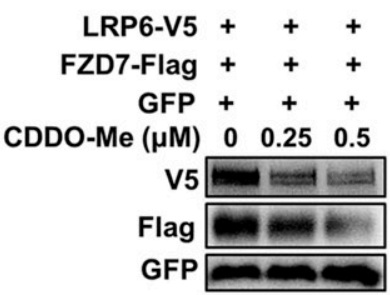

F

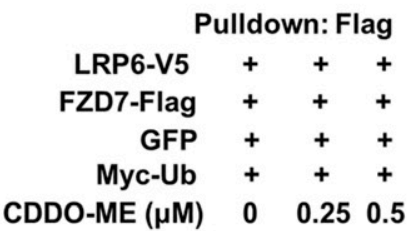

E

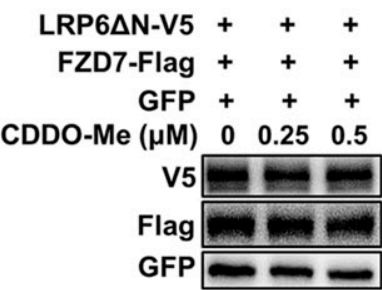

G
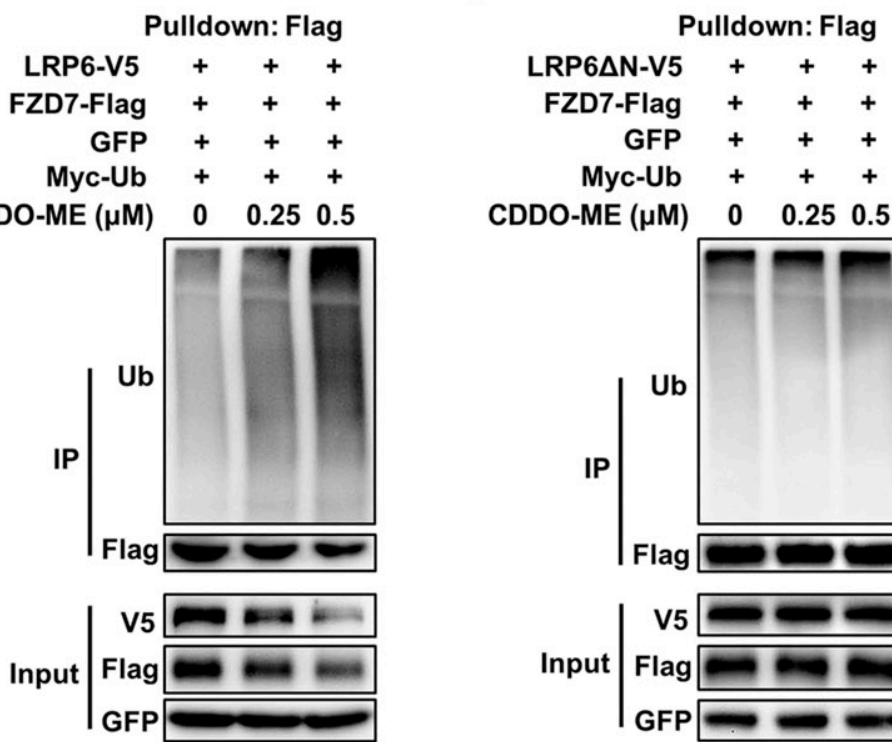

Fig. 5. CDDO-Me suppresses Wnt/ $\beta$-catenin signaling in an LRP6 ectodomain-dependent manner. (A and B). Hs578T (A) and MDA-MB-231 (B) cells were infected with lentivirus-mediated control shRNA (shC) or lentivirus-mediated shRNA directed toward LRP6 (shLRP6), and then cells were treated with the indicated amounts of CDDO-Me for 24 hours. The protein levels of LRP6 and FZD7 were detected by immunoblotting. (C) The SuperTOPFlash reporter was transfected into HEK293T cells together with empty vector or expression plasmids encoding LRP6 or LRP6 2 . The transfected cells were incubated with vehicle or the indicated concentrations of CDDO-Me for 24 hours. The luciferase values were normalized to $\beta$-gal activities. Each treatment was performed in six replicates. (D and E) HEK293T cells were transfected with expression plasmids encoding LRP6 (D) or LRP6 $\Delta \mathrm{N}$ (E) along with FZD7 and GFP expression vectors. After treatment with the indicated amounts of CDDO-Me for 24 hours, the protein levels of LRP6 and FZD7 were determined by immunoblotting. (F and G) HEK293T cells cotransfected with expression plasmids for FZD7-Flag, GFP, myc-Ub, and LRP6-V5 (F) or LRP6 6 N-V5 (G) were incubated with the indicated concentrations of CDDO-Me for 24 hours. Cell lysates were extracted and subjected to immunoprecipitation (IP) using anti-Flag M2-Sepharose followed by Western blotting using specific antibodies as indicated. The values are mean \pm S.E.M. Statistical analysis was conducted using one-way ANOVA followed by a Dunnett- $t$ test (C). CTRL, control. *P<0.05 compared with the vehicle control.

the YAP-stimulated Hippo reporter activity (Supplemental Fig. 1, R and S). These results suggest that CDDO-Me, CDDO, and OA may inhibit Wnt/ $\beta$-catenin signaling by a common mechanism.

CDDO-Me Inhibits Wnt/ $\beta$-Catenin Signaling in HEK293T Cells. To investigate the mechanism underlying Wnt-signaling inhibition by CDDO-Me, the Wnt/ $\beta$-catenin-signaling cascade was activated by either cotransfection with a Wnt1 expression plasmid or treatment with Wnt3A-CM in HEK293T cells. As shown in Fig. 2, Wnt1 expression or Wnt3A-CM increased phosphorylation of LRP6 and DVL2, resulting in increased protein levels of active $\beta$-catenin and total $\beta$-catenin (Fig. 2, A and B). Treatment with CDDO-Me markedly reduced the levels of phosphorylated LRP6, total LRP6, phosphorylated DVL2, active $\beta$-catenin, and total $\beta$-catenin in the cells transfected with Wnt1 (Fig. 2A) or treated with Wnt3A-CM (Fig. 2B). Similar to CDDO-Me, CDDO and OA also downregulated the levels of phosphorylated LRP6, total LRP6, phosphorylated DVL2, active $\beta$-catenin, and total $\beta$-catenin in the cells either transfected with Wnt1 expression vector (Supplemental Fig. 2, A and B) or treated with Wnt3A-CM (Supplemental Fig. 2, C and D). To further test the effect of CDDO-Me on protein levels of LRP6 and FZD, expression plasmids for LRP6-V5, FZD5-V5, or FZD7-FLAG were transfected into HEK293T cells. Treatment with CDDO-Me suppressed the levels of LRP6-V5, FZD5-V5, and FZD7-FLAG in a dose-dependent manner (Fig. 2C). These results suggest that CDDO-Me may induce the degradation of Wnt receptor complex components FZD and LRP6.

CDDO-Me Suppresses Wnt/B-Catenin Signaling in Breast Cancer Cells. To assess the effect of CDDO-Me on $\mathrm{Wnt} / \beta$-catenin signaling in breast cancer cells, three human breast cancer cell lines (Hs578T, MDA-MB-231, and MDAMB-468) were employed in this study. In these three cell lines, CDDO-Me significantly decreased the levels of phosphorylated LRP6 and total LRP6, FZD7, and phosphorylated DVL2 (Fig. $3, \mathrm{~A}-\mathrm{C}$ ). Both active $\beta$-catenin and total $\beta$-catenin levels were also reduced after CDDO-Me treatment. In contrast, CDDO-Me treatment had little effect on the expression of the noncanonical Wnt receptor, receptor tyrosine kinase-like orphan receptor 1 , and an irrelevant membrane protein, 


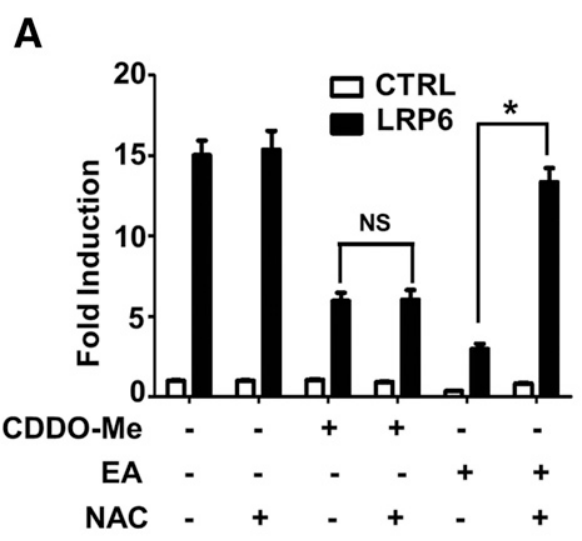

D

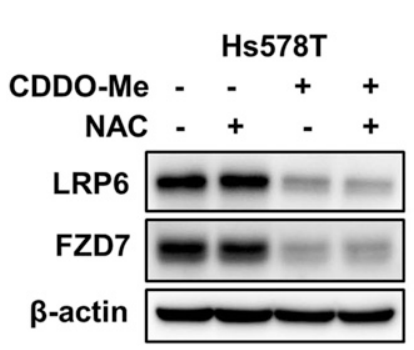

B
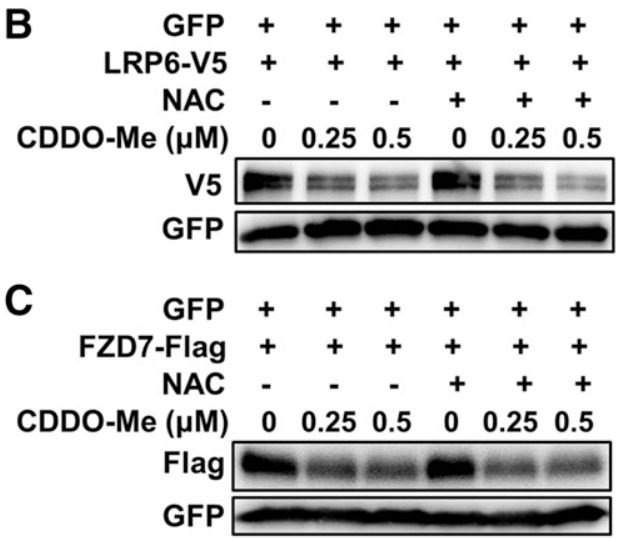

E

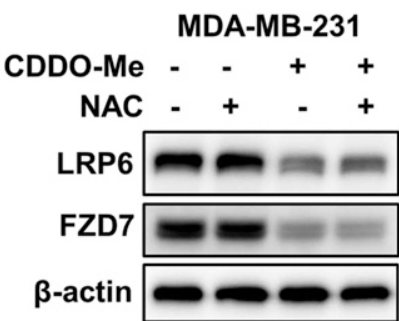

Fig. 6. CDDO-Me inhibits Wnt $/ \beta$-catenin signaling in an $\alpha, \beta$-unsaturated carbonyl moiety-independent manner. (A) The SuperTOPFlash reporter was transfected into HEK293T cells together with empty vector or LRP6 expression plasmid to activate $\mathrm{Wnt} / \beta$-catenin signaling. Then cells were treated with $0.4 \mu \mathrm{M}$ CDDO-Me or $70 \mu \mathrm{M}$ EA in the presence or absence of $1 \mathrm{mM}$ NAC. After 24 hours incubation, cells were lysed, and the luciferase activities were examined and normalized according to $\beta$-gal values. Each treatment was performed in six replicates. (B and $\mathrm{C}$ ) HEK293T cells were cotransfected with expression plasmids for GFP and LRP6V5 (B) or FZD7-Flag (C). Then cells were treated with the indicated concentrations of CDDO-Me in the presence or absence of $1 \mathrm{mM}$ NAC for 24 hours. Total protein was extracted and subjected to Western blotting using specific antibodies as indicated. (D and E) Hs578T (D) and MDAMB-231 (E) cells were treated with 0.4 $\mu \mathrm{M}$ CDDO-Me in the presence or absence of $1 \mathrm{mM}$ NAC for 24 hours. Total protein was extracted and subjected to Western blotting using specific antibodies as indicated. The values are mean \pm S.E.M. Statistical analysis was conducted using one-way ANOVA followed by a Dunnett- $t$ test (A). CTRL, control; NS, not significant. $* P<0.05$ compared as indicated groups.
ATP1A1, in Hs578T and MDA-MB-231 cells (Supplemental Fig. 3, A-D). Moreover, treatment with CDDO or OA also resulted in reduced levels of phosphorylated LRP6, total LRP6, phosphorylated DVL2, active $\beta$-catenin, and total $\beta$-catenin in MDA-MB-231 (Supplemental Fig. 4, A and B) and MDA-MB-468 (Supplemental Fig. 4, C and D) cells.

To further confirm the antagonistic effect of CDDO-Me on $\mathrm{Wnt} / \beta$-catenin signaling in breast cancer cells, real-time PCR was carried out to detect the expression of Wnt target genes, Fibronectin, Survivin, and Snail. Our results showed that CDDO-Me significantly decreased mRNA expression of Fibronectin, Survivin, and Snail in a concentration-dependent fashion in all three breast cancer cell lines (Fig. 3, D-F).

CDDO-Me Induces the Degradation of LRP6/FZD7 Receptor Complex. To investigate whether CDDOMe-induced downregulation of LRP6 and FZD7 was a consequence of decreased transcription, the real-time PCR assay was performed. Breast cancer Hs578T and MDA-MB-231 cells were treated with different concentrations of CDDO-Me for 24 hours. We did not observe any significant decrease in mRNA expression of LRP6 and FZD7 after CDDO-Me treatment in both cell lines (Fig. 4, A and B), indicating that the inhibitory effect of CDDO-Me on LRP6 and FZD7 was indeed independent of transcriptional regulation.

To determine the contributions of lysosomal and proteasomal pathways to CDDO-Me-induced degradation of LRP6 and FZD7, the lysosome inhibitor bafilomycin $\mathrm{A} 1$ and the proteasome inhibitor MG132 were employed. In Hs578T and MDAMB-231 cells, CDDO-Me-reduced expression of LRP6 and FZD7 was effectively restored by treatment with bafilomycin A1, but it was not restored by MG132 (Fig. 4, C and D). Consistently, we observed an increased lysosomal accumulation of FZD7 after CDDO-Me treatment in HEK293T cells
(Fig. 4E). These results indicate that the CDDO-Me-induced degradation of LRP6 and FZD7 is mediated by the lysosomal pathway rather than the proteasome pathway.

To determine whether CDDO-Me could increase LRP6 or FZD7 ubiquitination, HEK293T cells transfected with either LRP6-V5 or FZD7-FLAG expression plasmids together with expression vector for myc-ubiquitin (Ub) were treated with CDDO-Me, and the cell lysates were subjected to immunoprecipitation using anti-Ub and anti-V5 or antiFLAG antibodies. As shown in Fig. 4, F and G, CDDO-Me treatment increased the ubiquitination of LRP6 or FZD7 (Fig. 4, F and G).

CDDO-Me Induces FZD7 Degradation in an LRP6 Ectodomain-Dependent Manner. Interestingly, we noted that CDDO-Me failed to induce the degradation of FZD7 in LRP6-knockdown Hs578T cells (Fig. 5A). Similar results were observed in MDA-MB-231 cells (Fig. 5B). These results suggest that CDDO-Me-induced FZD7 degradation may be mediated by LRP6 in breast cancer cells.

To evaluate the role of LRP6 ectodomain in CDDOMe-induced FZD7 degradation, LRP6 $\Delta \mathrm{N}$ was constructed (Fig. 5C) (Su et al., 2018). HEK293T cells were transfected with expression plasmids encoding LRP6 or LRP $6 \Delta \mathrm{N}$. As expected, LRP $6 \Delta \mathrm{N}$ constitutively activated $\mathrm{Wnt} / \beta$-catenin signaling. CDDO-Me treatment did not exert any inhibitory effect on transcriptional activity induced by $L R P 6 \Delta N$ (Fig. 5C), suggesting the extracellular domain of LRP6 is required for CDDO-Me-induced degradation of FZD7. Consistent with this, CDDO-Me could not induce FZD7 degradation in the presence of LRP6 $\triangle \mathrm{N}$ (Fig. 5, D and E). Furthermore, in vitro ubiquitination experiments showed that CDDO-Me had little effect on the ubiquitination of FZD7 in the presence of LRP6 $\Delta \mathrm{N}$ (Fig. 5, F and G). 
A
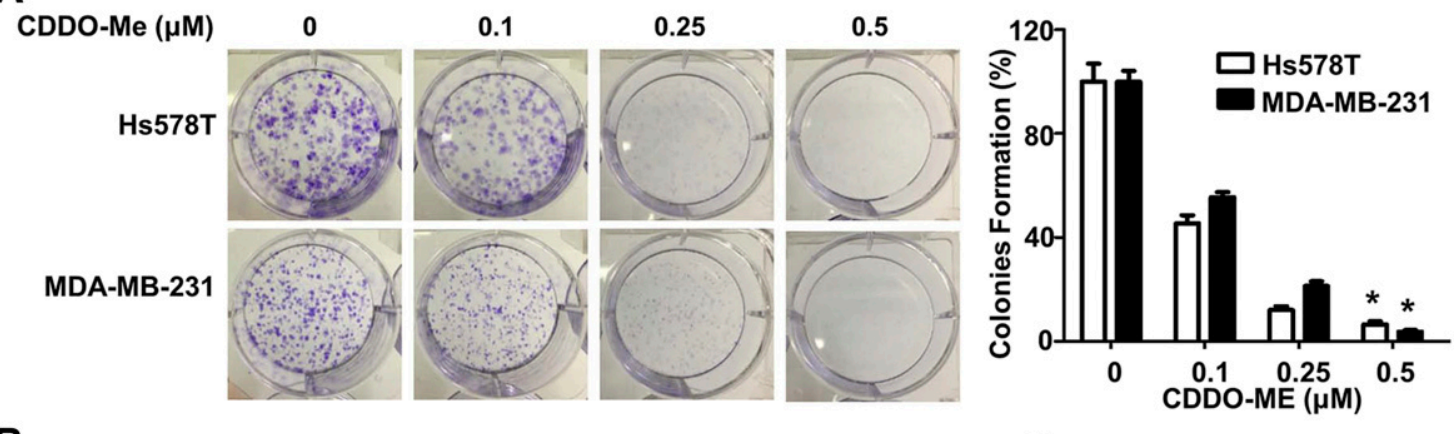

B
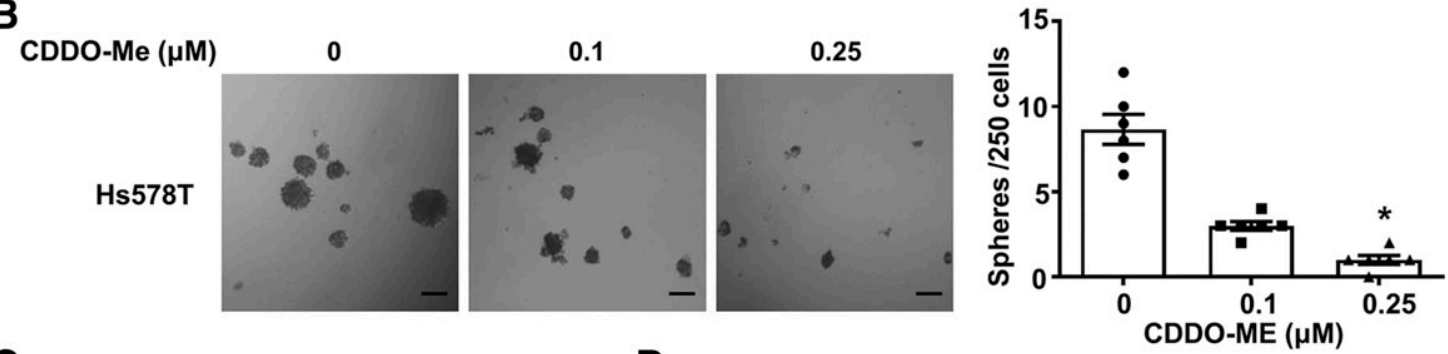

C

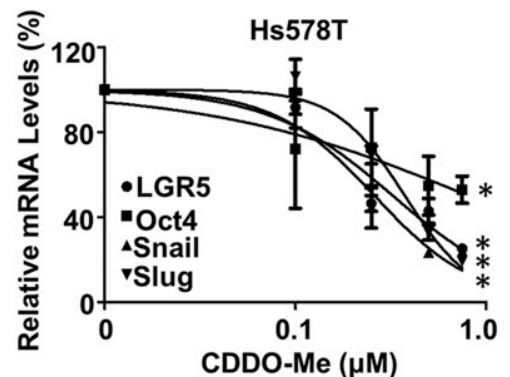

D

Hs578T

$\begin{array}{llllll}\text { CDDO-Me }(\mu \mathrm{M}) \quad 0 & 0.1 & 0.25 & 0.5 & 0.75\end{array}$

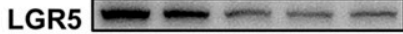

Oct4

Snail

Slug

$\beta$-actin

CDDO-Me $(\mu \mathrm{M})$

Fig. 7. CDDO-Me significantly suppresses proliferation and stemness in breast cancer cells. (A) Hs578T and MDA-MB-231 cells were treated with the indicated amounts of CDDO-Me for 7 days. Cells were then stained with crystal violet and photographed. The right panel: Graphical representation of quantitative data shows the relative number of colony formation per well. Each treatment was performed in six replicates. (B) Hs578T cells were cultured in Ultra-Low Attachment dishes to examine the ability of sphere formation in the absence or presence of the indicated concentrations of CDDOMe for 10 days. Right panel: Graphical representation of quantitative data shows the relative number of sphere formation per 250 cells. Each treatment concentration was performed in six replicates. (C) Hs578T cells were incubated with the indicated concentrations of CDDO-Me for 24 hours, and mRNA level of stemness marker genes LGR5, Oct4, Slug, and Snail were measured by real-time PCR. The data from three independent experiments are presented. (D) Hs578T cells were incubated with the indicated concentrations of CDDO-Me for 24 hours, and immunoblotting was performed to detect the protein levels of stemness marker genes LGR5, Oct4, Slug, and Snail. The values are mean \pm S.E.M. Statistical analysis was conducted using oneway ANOVA followed by a Dunnett- $t$ test (A-C). ${ }^{*} P<0.05$ compared with the vehicle control. Scale bar, $100 \mu \mathrm{m}$ (B).

CDDO-Me Inhibits the Wnt/ $\beta$-Catenin Pathway and Induces the Degradation of LRP6 and FZD7 in an $\alpha, \beta$-Unsaturated Carbonyl Moiety-Independent Manner. To assess whether the Wnt inhibitory effect of CDDO-Me is dependent on its $\alpha, \beta$-unsaturated carbonyl group, we examined the effect of CDDO-Me on Wnt/ $\beta$-catenin signaling and LRP6/FZD7 receptor complex in the presence of NAC. NAC possesses a reactive-free thiol group and can interact with the $\alpha, \beta$-unsaturated carbonyl moiety of CDDO-Me. Our results revealed that the presence of NAC had no effect on CDDO-Me-induced inhibition of SuperTOPFlash reporter activity (Fig. 6A), compoundinduced degradation of exogenous LRP6 and FZD7 in HEK293T cells (Fig. 6, B and C), and compound-induced degradation of endogenous LRP6 and FZD7 in Hs578T and MDA-MB-231 cells (Fig. 6, D and E). Meanwhile, we tested the effect of NAC on ethacrynic acid (EA)-mediated Wnt inhibition. EA contains an $\alpha, \beta$-unsaturated carbonyl unit and is a known antagonist of the $\mathrm{Wnt} / \beta$-catenin pathway (Jin et al., 2009; Lu et al., 2009). These results showed that
NAC could prevent EA-mediated effects on the Wnt/ $\beta$-catenin pathway (Fig. 6A).

CDDO-Me Inhibits the Wnt/ $\beta$-Catenin Pathway in a GSK3 $\beta$-Independent Manner. GSK3 $\beta$ is a serine/threonine kinase involved in multiple cellular responses, including modulation of $\beta$-catenin stability. Its kinase activity is negatively regulated by phosphorylation of GSK3 $\beta$ at Ser9. A previous study reported that apoptotic doses of CDDO-Me $(1-2.5 \mu \mathrm{M})$ could induce inactivating phosphorylation at Ser9 of GSK3 $\beta$ in prostate cancer cells (Vene et al., 2008). Our results showed that CDDO-Me at nanomolar or low micromolar concentrations (100-500 nM) had little effect on phosphorylation at Ser9 of GSK3 $\beta$ in Hs578T cells (Supplemental Fig. 5), whereas increased phosphorylation at Ser9 of GSK3 $\beta$ was observed after treatment with $1 \mu \mathrm{M}$ CDDO-Me in Hs578T cells. These results suggest that the antagonistic effect of CDDO-Me on Wnt/ $\beta$-catenin signaling was independent of GSK3 $\beta$.

CDDO-Me Represses Cell Viability, Proliferation, Colony Formation, and Stemness in Breast Cancer Cells. We tested the cytotoxic effect of CDDO-Me on breast 
A

A

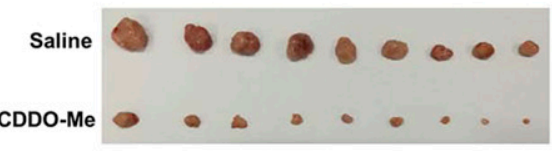

D

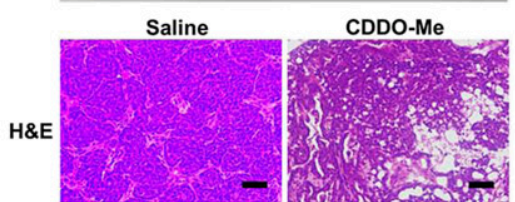

E

Saline
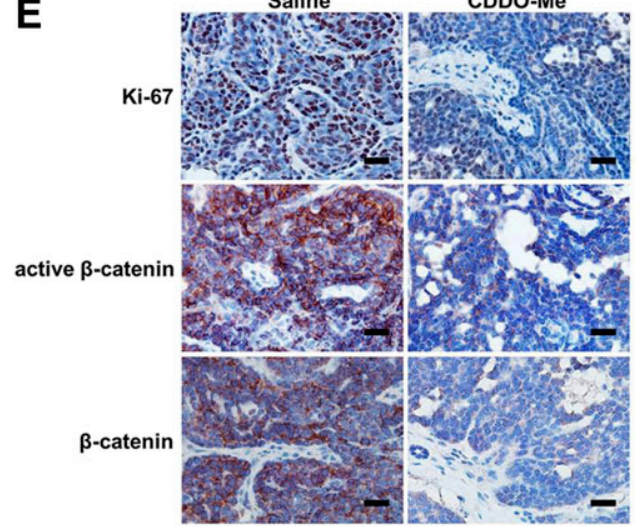

B

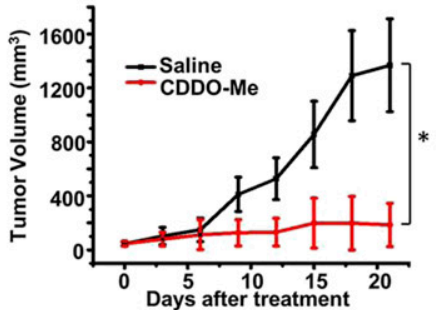

$\mathbf{F}$

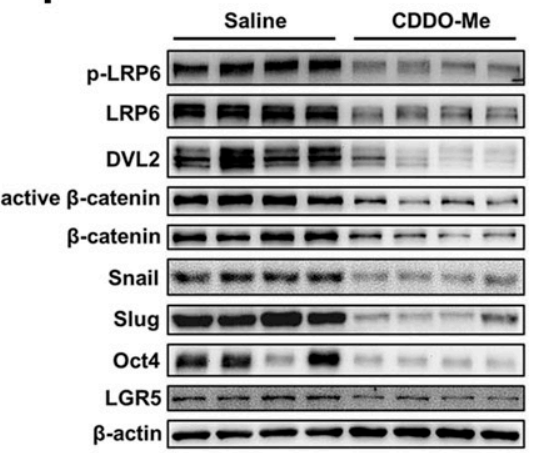

C

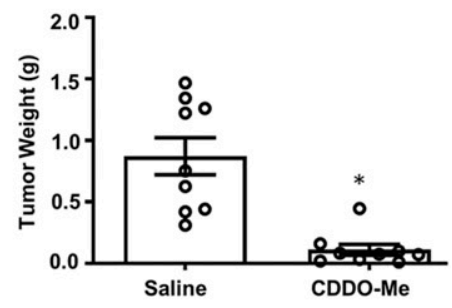

$\mathbf{G}$

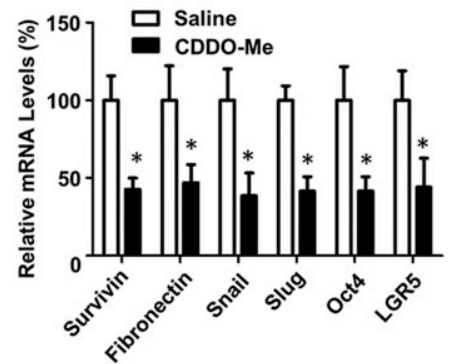

Fig. 8. CDDO-Me inhibits the Wnt/ $\beta$-catenin signaling pathway in a murine xenograft model bearing Wnt1-driven murine mammary tumor. Breast tumor fragments from MMTV-Wnt1 mice were implanted subcutaneously into BALB/c nude mice. When the tumors reached $\sim 50 \mathrm{~mm}^{3}$, the mice were randomly divided into two groups and treated with the vehicle or $5 \mathrm{mg} / \mathrm{kg}$ CDDO-Me in vehicle twice weekly by intraperitoneal injection. At the endpoint, $\mathrm{CO}_{2}$ euthanasia were performed. Then tumors were excised, weighed, and photographed. (A) Image of tumors. (B) Tumor growth curve. (C) Tumor weight. (D) Hematoxylin and eosin (H\&E) staining of tumor section. (E) Immunohistochemistry of Ki-67, active $\beta$-catenin (ABC), and total $\beta$-catenin. (F) Total protein was extracted from tumor samples, and immunoblotting was performed to detect the protein levels of phosphorylated LRP6 (Ser1490), total LRP6, FZD7, DVL2, active $\beta$-catenin, total $\beta$-catenin, LGR5, Oct4, Slug, and Snail. (G) Total RNA was extracted from tumor samples and real-time PCR was used to determine mRNA levels of Wnt target genes and stemness-associated genes $(n=8)$. The values are mean \pm S.E.M. Statistical analysis was performed with Student's $t$ test (C and G) and one-way ANOVA followed by a Dunnett- $t$ test $(\mathrm{B})$. $* P<0.05$ compared with the vehicle control. Scale bar, $100 \mu \mathrm{m}$ (D). Scale bar, $50 \mu \mathrm{m}$ (E).

cancer cells using 3-(4,5-dimethylthiazol-2-yl)-2,5-diphenyltetrazolium bromide assay. Consistent with results from earlier studies (Bishayee et al., 2011; El-Ashmawy et al., 2014), CDDO-Me decreased the viability of breast cancer cells, with $\mathrm{IC}_{50}$ values at 48 hours of $0.51 \mu \mathrm{M}$ in Hs578T cells, $0.42 \mu \mathrm{M}$ in MDA-MB-231 cells, and $0.55 \mu \mathrm{M}$ in MDA-MB468 cells (Supplemental Fig. 6, A and B). BrdU incorporation assay was performed to evaluate the effect of CDDO-Me on cellular proliferation. Treatment with CDDO-Me caused a dose-dependent decrease in BrdU incorporation in Hs578T, MDA-MB-231, and MDA-MB-468 cells (Supplemental Fig. 6C). We further assessed the colonyforming ability of breast cancer cells using cell colony formation assay. As shown in Fig. 7A, CDDO-Me markedly repressed the clonogenic ability of Hs578T and MDA-MB231 cells (Fig. 7A).

Since Wnt $/ \beta$-catenin signaling plays a critical role in cancer stem cell (CSC) maintenance, we used the sphere formation assay in vitro to evaluate the effect of CDDO-Me on CSC selfrenewal potential. Nanomolar concentrations of CDDO-Me significantly reduced tumorsphere formation in Hs578T cells (Fig. 7B). Given the role of Wnt/ $\beta$-catenin signaling in the regulation of expression of CSC marker genes, we examined the effect of CDDO-Me on the expression of CSC marker genes LGR5, Oct4, Snail, and Slug. Real-time PCR and immunoblotting revealed that CDDO-Me treatment decreased the mRNA and protein levels of CSC markers LGR5, Oct4, Snail, and Slug (Fig. 7, C and D).
CDDO-Me Inhibits the Growth of MMTV-Wnt1 Tumor Allografts via the Downregulation of Wnt/ $\boldsymbol{\beta}$-Catenin Signaling. We tested the antitumor efficacy of CDDO-Me against MMTV-Wnt1 tumor allografts. The primary MMTV-Wnt1 tumors were fragmented and subcutaneously implanted into $\mathrm{BALB} / \mathrm{c}$ nude mice. Once the tumors reached approximately $50 \mathrm{~mm}^{3}$, mice were intraperitoneally injected with either vehicle or $5 \mathrm{mg} / \mathrm{kg}$ CDDO-Me twice a week for 3 weeks. CDDO-Me administration arrested tumor growth (Fig. 8, A-C). The treatment regimen was well-tolerated and did not cause significant weight loss (unpublished data). Histologic analyses showed that CDDO-Me treatment reduced tumor cell density and proliferation, as assessed by Ki-67 staining (Fig. 8, D and E).

To investigate whether CDDO-Me-induced inhibition of tumor growth was accompanied with the suppression of Wnt/ $\beta$-catenin signaling, the tumor tissues from the control and treatment groups were examined using immunohistochemical staining, immunoblot analyses, and real-time PCR. Administration of CDDO-Me noticeably reduced the expression of active and total $\beta$-catenin (Fig. 8E). The CDDO-Me-treated tumors exhibited markedly decreased levels of phosphorylated and total LRP6, phosphorylated and unphosphorylated DVL2, and CSC-related markers Snail, Slug, Oct4, and LGR5 compared with the vehicle-treated tumors (Fig. 8F). Moreover, the expression levels of Wnt target genes Fibronectin, Survivin, Snail, Slug, Oct4, and LGR5 were significantly reduced in tumors from mice treated with CDDO-Me (Fig. 8G). 

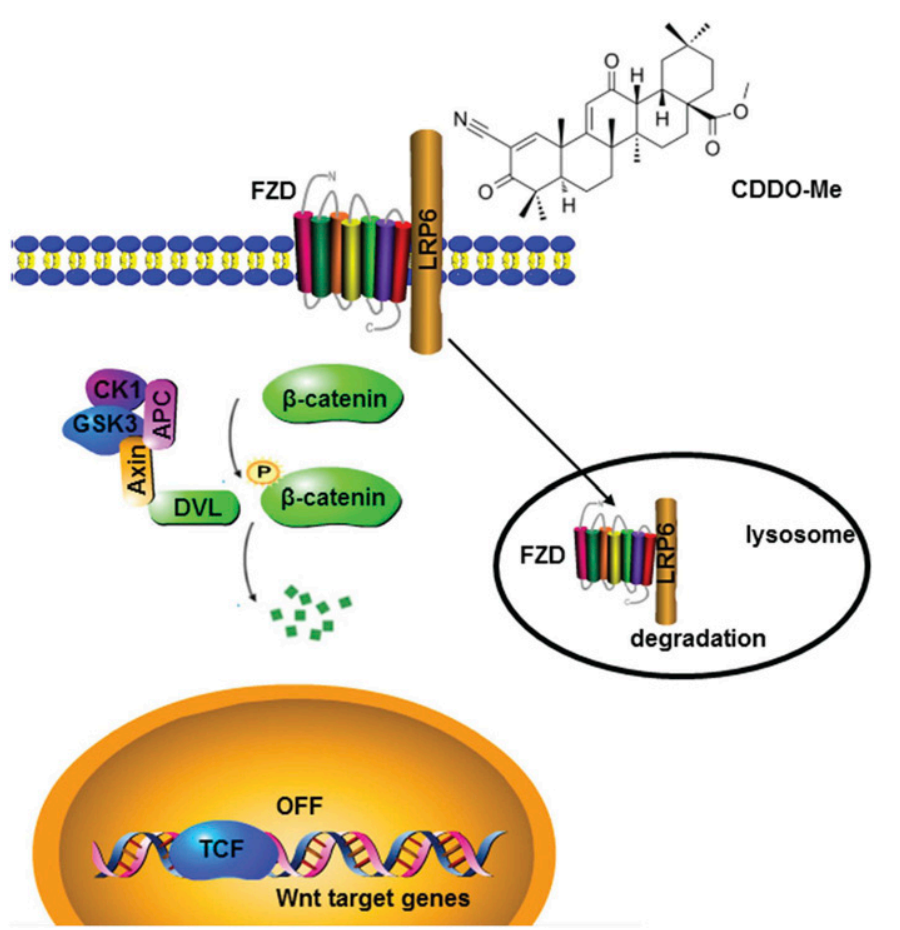

Fig. 9. A schematic summary for the inhibition of the Wnt/ $\beta$-catenin signaling pathway by CDDO-Me. CDDO-Me induces the degradation and ubiquitination of LRP6/FZD7 receptor complex via the lysosomal pathway, thereby decreasing the levels of phosphorylated DVL and active $\beta$-catenin, eventually resulting in the downregulation of Wnt target genes. CK1, casein kinase 1; TCF, T-cell factor.

\section{Discussion}

Although genetic mutations in the genes encoding intracellular components of Wnt signaling are rare, aberrant activation of this signaling pathway is frequently observed in human breast cancer. Accumulating evidence indicates that dysregulation of cell-surface Wnt-signaling components may contribute to constitutive activation of this pathway in breast cancer (Lamb et al., 2013; Pohl et al., 2017; Zhan et al., 2017). The Wnt-signaling coreceptor LRP6 has been shown to be upregulated in a subset of human breast cancer tissues and cell lines (Liu et al., 2010). Knockdown of LRP6 in breast cancer cells significantly decreased Wnt/ $\beta$-catenin signaling, cell proliferation, and tumor growth in vivo (Liu et al., 2010). Notably, overexpression of LRP6 in mammary epithelial cells driven by the MMTV promoter is sufficient to induce mammary gland hyperplasia (Zhang et al., 2010). The Wnt receptor FZD7 also plays a critical role in cell proliferation in triple-negative breast cancer. Yang et al. (2011) reported that FZD7 was overexpressed in triple-negative breast cancer tissues and cell lines. Downregulation of FZD7 in breast cancer cells resulted in impaired cell growth and tumor transformation (Yang et al., 2011). In vivo studies revealed that FZD7 shRNA induced growth retardation via blocking the $\mathrm{Wnt} / \beta$-catenin signaling pathway (Yang et al., 2011). These studies prove the concept that LRP6 and FZD7 may serve as potential therapeutic targets for the treatment of breast cancer. Inhibition of either FZD7 or LRP6 may block Wnt signaling and suppress Wnt signal-mediated cell proliferation. In this study, we demonstrated that CDDO-Me could inhibit Wnt/ $\beta$-catenin signaling through targeting LRP6/FZD7 complex in breast cancer cells. This compound-induced lysosomal degradation of LRP6/FZD7 complex, resulting in a decrease in DVL phosphorylation and downregulation of active $\beta$-catenin and total $\beta$-catenin protein. Furthermore, CDDO-Me suppressed the growth of MMTVWnt1 tumor allografts via the downregulation of Wnt $/ \beta$-catenin signaling. Taken together, our results define a novel mechanism for anticancer action of CDDO-Me.

CDDO-Me, an $\alpha, \beta$-unsaturated carbonyl compound, reacts with glutathione, leading to the formation of Michael adducts between CDDO-Me and reactive nucleophiles on target proteins (Wang et al., 2014a; Chaudhari and Ravanan, 2018). This compound has been shown to inhibit the NF- $\kappa \mathrm{B}$ pathway by direct alkylation of Cys179 in the inhibitor of $\kappa \mathrm{B}$ kinase $\beta$ activation loop (Ahmad et al., 2006). Ahmad et al. (2008) reported that CDDO-Me could block activation of the JAK1/STAT3 pathway by forming cysteine adducts with both JAK1 and STAT3. It is possible that CDDO-Me may induce the degradation of LRP6/FZD7 receptor complex in an $\alpha, \beta$-unsaturated carbonyl moiety-dependent way. NAC, a pharmacological precursor of L-cysteine, was used to test this hypothesis. The chemical reaction between NAC and CDDO-Me should form NAC-CDDO-Me adduct through Michael addition reaction, which may result in an abrogation of CDDO-Me-induced degradation. However, pretreatment with NAC had no effect on CDDO-Me-induced degradation of LRP6/FZD7 receptor complex, indicating that CDDO-Me may induce the degradation of LRP6/FZD7 receptor complex through an $\alpha, \beta$-unsaturated carbonyl moiety-independent way. Future studies are needed to investigate the mechanism underlying CDDO-Me-induced degradation of LRP6/FZD7 receptor complex.

CSCs are a small population of tumor cells responsible for tumor initiation, recurrence, metastasis, and drug resistance in multiple tumor types (Agliano et al., 2017; Phi et al., 2018). Several key developmental signaling pathways, such as Wnt, Notch, and Hedgehog, are required for maintaining the stemness of CSCs. In human breast cancer, the CD $44^{+} \mathrm{CD} 24^{- \text {llow }}$ Lineage $^{-}$cells were originally identified as CSCs. As few as 100 of these cells were able to form tumors in the nonobese diabetic/severe combined immunodeficiency mice (Al-Hajj et al., 2003). Other cellular surface markers, such as CD133, CD166, epithelial cell adhesion molecule (EpCAM), and aldehyde dehydrogenase, have also been used for the isolation of breast CSCs (Xia, 2014; Saeg and Anbalagan, 2018). CDDOIm is an imidazole-containing oleanane triterpenoid. This compound has been shown to inhibit the tumorsphere formation of breast cancer SUM159 cells. Moreover, CDDO-Im could suppress the $\mathrm{CD} 44^{+} / \mathrm{CD} 24^{-1 \text { low }} /$ epithelial cell adhesion molecule $(\mathrm{EpCAM})^{+}$cells in SUM159 tumorspheres (So et al., 2014). The present study demonstrates that CDDO-Me markedly inhibited tumorsphere formation in breast cancer Hs578T cells and resulted in the downregulation of CSC marker genes LGR5, Oct4, Snail, and Slug via antagonizing $\mathrm{Wnt} / \beta$-catenin signaling. Importantly, the administration of CDDO-Me reduced the expression of CSC marker genes LGR5, Oct4, Snail, and Slug in a Wnt1-driven murine mammary tumor model. These results indicate that CDDOMe may exhibit its anti-breast cancer activity through targeting breast CSCs.

In conclusion, CDDO-Me could directly bind to the extracellular domain of LRP6 and induce lysosomal degradation of LRP6/FZD7 receptor complex, resulting in the downregulation of Wnt/ $\beta$-catenin signaling in vitro and in vivo (Fig. 9). In 
an MMTV-Wnt1 xenograft model, CDDO-Me significantly suppressed tumor growth via the inhibition of $\mathrm{Wnt} / \beta$-catenin signaling. These results indicate that the anti-breast cancer activity of CDDO-Me is associated with its antagonistic effect on the $\mathrm{Wnt} / \beta$-catenin signaling cascade.

\section{Acknowledgments}

The authors would like to acknowledge the support provided by International Science and Technology Cooperation: Carson Cancer Stem Cell Vaccines R \& D Center, Shenzhen University.

\section{Authorship Contributions}

Participated in research design: Zhou, Wang, Lu.

Conducted experiments: Zhou, Wang, Yu, Xiong, Fan, Lyu, Su, Song, Liu, Sun.

Contributed new reagents or analytic tools: Song.

Performed data analysis: Zhou, Wang, Lyu, Lu.

Wrote or contributed to the writing of the manuscript: Zhou, Wang, Lu.

\section{References}

Agliano A, Calvo A, and Box C (2017) The challenge of targeting cancer stem cells to halt metastasis. Semin Cancer Biol 44:25-42.

Ahmad R, Raina D, Meyer C, Kharbanda S, and Kufe D (2006) Triterpenoid CDDOMe blocks the NF-kappaB pathway by direct inhibition of IKKbeta on Cys-179. J Biol Chem 281:35764-35769.

Ahmad R, Raina D, Meyer C, and Kufe D (2008) Triterpenoid CDDO-methyl ester inhibits the Janus-activated kinase-1 (JAK1)--> signal transducer and activator of transcription-3 (STAT3) pathway by direct inhibition of JAK1 and STAT3. Cancer Res 68:2920-2926.

Ai Y, Kang F, Huang Z, Xue X, Lai Y, Peng S, Tian J, and Zhang Y (2015) Synthesis of CDDO-amino acid-nitric oxide donor trihybrids as potential antitumor agents against both drug-sensitive and drug-resistant colon cancer. J Med Chem $\mathbf{5 8}$ $2452-2464$.

Al-Hajj M, Wicha MS, Benito-Hernandez A, Morrison SJ, and Clarke MF (2003) Prospective identification of tumorigenic breast cancer cells. Proc Natl Acad Sci USA 100:3983-3988.

Ayeleso TB, Matumba MG, and Mukwevho E (2017) Oleanolic acid and its derivatives: biological activities and therapeutic potential in chronic diseases. Molecules 22:E1915.

Bishayee A, Ahmed S, Brankov N, and Perloff M (2011) Triterpenoids as potential agents for the chemoprevention and therapy of breast cancer. Front Biosci 16 $980-996$.

Chaudhari N and Ravanan P (2018) Bardoxolone methyl induces neuritogenesis in Neuro2a cells. Pharmacol Rep 70:730-736.

Clevers H and Nusse R (2012) Wnt/3-catenin signaling and disease. Cell 149 1192-1205.

Deeb D, Gao X, Jiang H, Dulchavsky SA, and Gautam SC (2009) Oleanane triterpenoid CDDO-Me inhibits growth and induces apoptosis in prostate cancer cells by independently targeting pro-survival Akt and mTOR. Prostate 69:851-860.

El-Ashmawy M, Delgado O, Cardentey A, Wright WE, and Shay JW (2014) CDDOMe protects normal lung and breast epithelial cells but not cancer cells from radiation. PLoS One 9:e115600

Jin G, Lu D, Yao S, Wu CC, Liu JX, Carson DA, and Cottam HB (2009) Amide derivatives of ethacrynic acid: synthesis and evaluation as antagonists of Wnt/betacatenin signaling and CLL cell survival. Bioorg Med Chem Lett 19:606-609.

Krishnamurthy N and Kurzrock R (2018) Targeting the Wnt/beta-catenin pathway in cancer: update on effectors and inhibitors. Cancer Treat Rev 62:50-60.

Lamb R, Ablett MP, Spence K, Landberg G, Sims AH, and Clarke RB (2013) Wnt pathway activity in breast cancer sub-types and stem-like cells. PLoS One 8 e67811.

Liu CC, Prior J, Piwnica-Worms D, and Bu G (2010) LRP6 overexpression defines a class of breast cancer subtype and is a target for therapy. Proc Natl Acad Sci USA 107:5136-5141.

Lu D, Liu JX, Endo T, Zhou H, Yao S, Willert K, Schmidt-Wolf IG, Kipps TJ, and Carson DA (2009) Ethacrynic acid exhibits selective toxicity to chronic lymphocytic leukemia cells by inhibition of the Wnt/beta-catenin pathway. PLoS One 4:e8294.

MacDonald BT, Tamai K, and He X (2009) Wnt/beta-catenin signaling: components, mechanisms, and diseases. Dev Cell 17:9-26.

Mirabelli CK, Nusse R, Tuveson DA, and Williams BO (2019) Perspectives on the role of Wnt biology in cancer. Sci Signal 12

Nusse R and Clevers H (2017) Wnt/ $\beta$-catenin signaling, disease, and emerging therapeutic modalities. Cell 169:985-999.

Phi LTH, Sari IN, Yang YG, Lee SH, Jun N, Kim KS, Lee YK, and Kwon HY (2018) Cancer stem cells (CSCs) in drug resistance and their therapeutic implications in cancer treatment. Stem Cells Int 2018:5416923.

Pohl SG, Brook N, Agostino M, Arfuso F, Kumar AP, and Dharmarajan A (2017) Wnt signaling in triple-negative breast cancer. Oncogenesis 6:e310.

Saeg F and Anbalagan M (2018) Breast cancer stem cells and the challenges of eradication: a review of novel therapies. Stem Cell Investig 5:39.

So JY, Lin JJ, Wahler J, Liby KT, Sporn MB, and Suh N (2014) A synthetic triterpenoid CDDO-Im inhibits tumorsphere formation by regulating stem cell signaling pathways in triple-negative breast cancer. PLoS One 9:e107616.

Su Z, Song J, Wang Z, Zhou L, Xia Y, Yu S, Sun Q, Liu SS, Zhao L, Li S, et al. (2018) Tumor promoter TPA activates Wnt/B-catenin signaling in a casein kinase 1dependent manner. Proc Natl Acad Sci USA 115:E7522-E7531.

To C, Ringelberg CS, Royce DB, Williams CR, Risingsong R, Sporn MB, and Liby KT (2015) Dimethyl fumarate and the oleanane triterpenoids, CDDO-imidazolide and CDDO-methyl ester, both activate the Nrf2 pathway but have opposite effects in the $\mathrm{A} / \mathrm{J}$ model of lung carcinogenesis. Carcinogenesis 36:769-781.

Venè R, Larghero P, Arena G, Sporn MB, Albini A, and Tosetti F (2008) Glycogen synthase kinase 3 beta regulates cell death induced by synthetic triterpenoids. Cancer Res 68:6987-6996.

Wang XY, Zhang XH, Peng L, Liu Z, Yang YX, He ZX, Dang HW, and Zhou SF (2017) Bardoxolone methyl (CDDO-Me or RTA402) induces cell cycle arrest, apoptosis and autophagy via PI3K/Akt/mTOR and p38 MAPK/Erk1/2 signaling pathways in K562 cells. Am J Transl Res 9:4652-4672.

Wang YY, Yang YX, Zhao R, Pan ST, Zhe H, He ZX, Duan W, Zhang X, Yang T, Qiu $J X$, et al. (2015) Bardoxolone methyl induces apoptosis and autophagy and inhibits epithelial-to-mesenchymal transition and stemness in esophageal squamous cancer cells. Drug Des Devel Ther 9:993-1026.

Wang YY, Yang YX, Zhe H, He ZX, and Zhou SF (2014a) Bardoxolone methyl (CDDO$\mathrm{Me})$ as a therapeutic agent: an update on its pharmacokinetic and pharmacodynamic properties. Drug Des Devel Ther 8:2075-2088.

Wang YY, Zhe H, and Zhao R (2014b) Preclinical evidences toward the use of triterpenoid CDDO-Me for solid cancer prevention and treatment. Mol Cancer 13:30.

Wang Z, Li B, Zhou L, Yu S, Su Z, Song J, Sun Q, Sha O, Wang X, Jiang W, et al. (2016) Prodigiosin inhibits Wnt/B-catenin signaling and exerts anticancer activity in breast cancer cells. Proc Natl Acad Sci USA 113:13150-13155.

Willert K, Shibamoto S, and Nusse R (1999) Wnt-induced dephosphorylation of axin releases beta-catenin from the axin complex. Genes Dev 13:1768-1773.

Xia P (2014) Surface markers of cancer stem cells in solid tumors. Curr Stem Cell Res Ther 9:102-111.

Xu D, Chen L, Chen X, Wen Y, Yu C, Yao J, Wu H, Wang X, Xia Q, and Kong X (2017) The triterpenoid CDDO-imidazolide ameliorates mouse liver ischemia-reperfusion injury through activating the Nrf2/HO-1 pathway enhanced autophagy. Cell Death Dis 8:e2983

Yadav VR, Prasad S, Sung B, Kannappan R, and Aggarwal BB (2010) Targeting inflammatory pathways by triterpenoids for prevention and treatment of cancer. Toxins (Basel) 2:2428-2466.

Yang L, Wu X, Wang Y, Zhang K, Wu J, Yuan YC, Deng X, Chen L, Kim CC, Lau S, et al. (2011) FZD7 has a critical role in cell proliferation in triple negative breast cancer. Oncogene 30:4437-4446.

Zagorski JW, Turley AE, Freeborn RA, VanDenBerg KR, Dover HE, Kardell BR, Liby KT, and Rockwell CE (2018) Differential effects of the Nrf2 activators tBHQ and CDDO-Im on the early events of T cell activation. Biochem Pharmacol 147:67-76.

Zhan T, Rindtorff N, and Boutros M (2017) Wnt signaling in cancer. Oncogene 36: 1461-1473

Zhang J, Li Y, Liu Q, Lu W, and Bu G (2010) Wnt signaling activation and mammary gland hyperplasia in MMTV-LRP6 transgenic mice: implication for breast cancer tumorigenesis. Oncogene 29:539-549.

Zhong Z and Virshup DM (2020) Wnt signaling and drug resistance in cancer. Mol Pharmacol 97:72-89.

Address correspondence to: Desheng Lu, Department of Pharmacology, Shenzhen University Health Science Center, Shenzhen, Guangdong 518060 China. E-mail: delu@szu.edu.cn 